\title{
Integrative Computational Frameworks for Multiscale Digital Human Modeling and Simulation ${ }^{\star}$
}

\author{
Richard C. Ward ${ }^{1}$, Line C. Pouchard ${ }^{2}$, and James J. Nutaro ${ }^{1}$ \\ 1 Computational Sciences and Engineering Division, Oak Ridge National Laboratory, \\ P.O. Box 2008, Bethel Valley Road, Oak Ridge, Tennessee, USA \\ $\{$ wardrc1, nutarojj\}@ornl.gov \\ 2 Computer Sciences and Mathematics Division, Oak Ridge National Laboratory, \\ P.O. Box 2008, Bethel Valley Road, Oak Ridge, Tennessee, USA \\ pouchardlc@ornl.gov
}

\begin{abstract}
Integrated digital human modeling has seen increased interest over the last decade. We describe two efforts to develop computational frameworks for digital human modeling and describe the progress toward understanding the requirements for implementation. Both projects addressed data repository, computational environment, and visualization of results. But neither environment was a true problem-solving environment in that integration of computations with visualization capabilities was limited or absent. We detail the development of the computational environments for each effort and then provide proposals for improving the integration of the various components of a future "Digital Human" computational environment.
\end{abstract}

\section{Introduction}

Integrated digital human modeling has seen increased interest over the last decade. Here we report on two efforts; the first led by Oak Ridge National Laboratory (ORNL) and a more recent larger effort in which ORNL played a role.

\footnotetext{
* Portions of this research were supported by a grant from the Defense Advanced Research Projects Agency, executed by the U.S. Army Medical Research and Materiel Command/TATRC Cooperative Agreement, Contract W81XWH-04-2-0012. Portions of this research were sponsored by the Laboratory Directed Research and Development Program of Oak Ridge National Laboratory, managed by UT-Battelle, LLC, for the U. S. Department of Energy under Contract No. DE-AC05-00OR22725.

The submitted manuscript has been authored by the U.S. Department of Energy, Office of Science of the Oak Ridge National Laboratory, managed for the U.S. DOE by UT-Battelle, LLC, under contract No. DE-AC05-00OR22725. Accordingly, the U.S. Government retains a non-exclusive, royalty-free license to publish or reproduce the published form of this contribution, or allow others to do so, for U.S. Government purpose.
}

Approved for Public Release, Distribution Unlimited. 
The first effort, Virtual Human (VH), culminated in the development of a Webbased, Java client/server environment within which some simple cardiovascular circuit models and various other compartment models were incorporated. The second effort, the Defense Advance Research Projects Agency (DARPA)-funded Virtual Soldier (VS) Project, was a much more substantial effort to address human modeling and predict the consequences of a soldier being wounded on the battlefield. A more comprehensive project, this second effort addressed the development of an integrated data repository, computational environment, and visualization of results and predictions in a concept referred to as the holographic medical electronic representation (Holomer) [1].

Both these projects contributed significantly to our understanding of how a computational framework can be built to address virtual human simulation. However, the degree of integration of various components of the environment left much to be desired. Neither environment fully integrated computations with other elements of the environment; continued work must be done to bring the design of digital human modeling environments in line with present thinking in Service-Oriented Architecture (SOA) (http://www.service-architecture.com/) for flexibility of integrating data, computational, and visualization components.

Finally, we describe some concepts that might facilitate full integration and provide an end-to-end capability for data acquisition, model computation, and display of results and predictions.

\section{Virtual Human}

The concept of a VH was developed during 1996-2000. The VH was a "concept...to combine models and data to build a comprehensive computational capability for simulating the function as well as the structure of the human body and allow trauma simulations as well as many other applications" 2. In the fall of 1999 a workshop was held in Rockville, Maryland under ORNL auspices. The general interest in the concept and enthusiasm for developing a VH, as well as discussions in the Architecture breakout group, laid a foundation for subsequent design and development of a computational framework.

\subsection{VH Computational Environment}

ORNL then initiated an effort to develop a prototype VH computational environment. We chose to develop a Web-delivered environment using Java Remote Method Invocation (RMI) with the human anatomical geometry defined using Virtual Reality Modeling Language (VRML) [9] [8]. The software underlying the computational environment was derived from a generic client-server simulation framework 3. The environment allowed for integration of models written in different programming languages integrated using Java Native Interface (JNI). Remote steering of the computation was also incorporated into the $\mathrm{VH}$ computational environment. The user interface is displayed in Fig. 1, showing windows with the anatomical geometry, a circuit model for the left side of the heart, and 


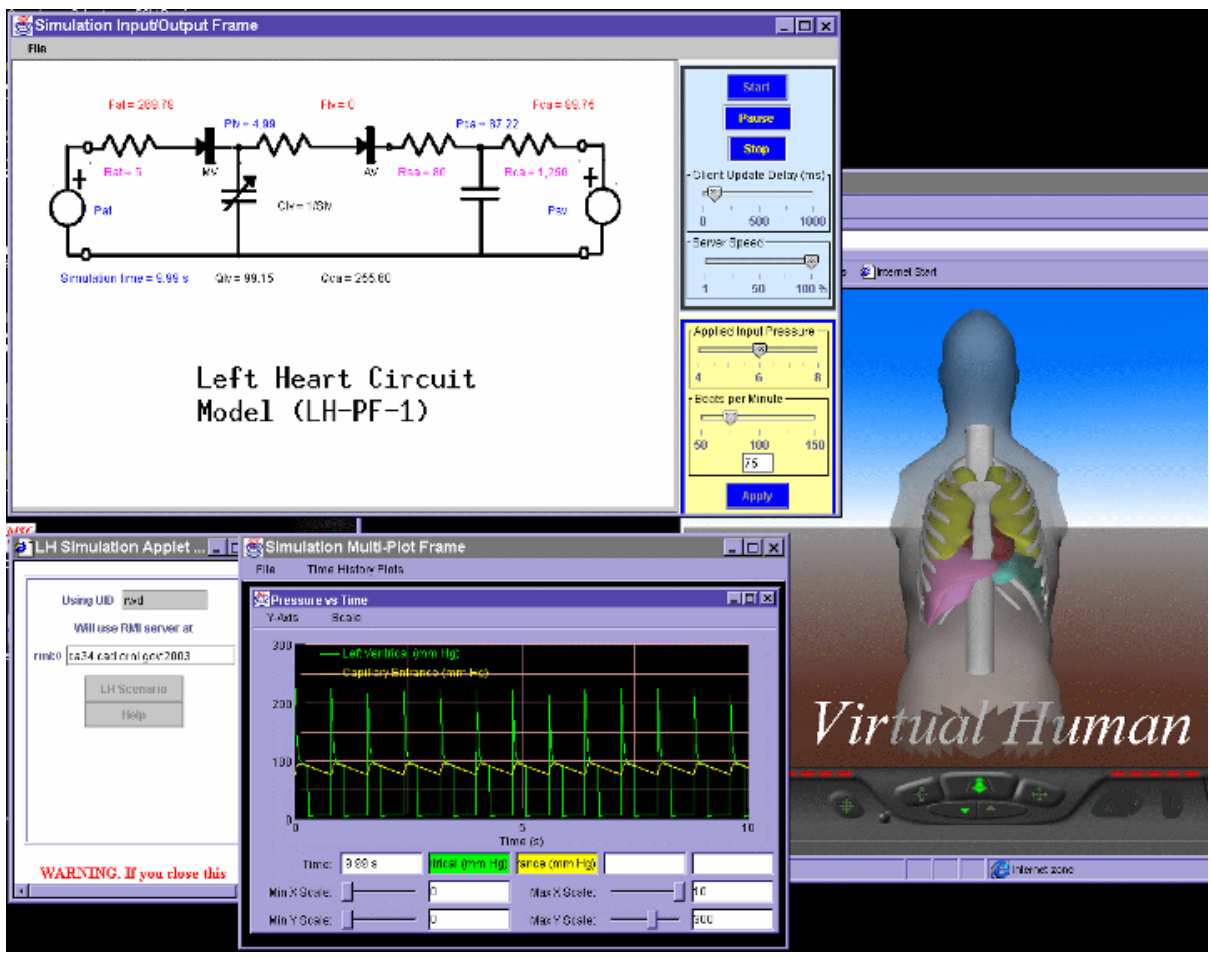

Fig. 1. Virtual Human User Interface

the physiological response of that model. The anatomical geometry used here was a portion of the National Library of Medicine Visible Human male data converted to VRML format.

\subsection{PhysioML}

The most significant development, however, was to create PhysioML, a physiological Extensible Markup Language (XML) to support model description [9]. PhysioML contains tags to describe the model parameters and variables and associated units. Initial conditions are also defined and the user can select particular variables which can be altered during the computation (computational steering). Finally, PhysioML has tags to describe the user interface including placement of parameters and variables on the interface and graphical representation of the results of the simulation.

Thus, PhysioML incorporates the parameters for the physiological model equations, control of variables for steering the computation, and tags for display of the results in the user interface. While other XML languages such as SBML (http://sbml.org/) and cellML (http://www.cellml.org/) incorporated the first concept, PhysioML is unique in providing a capability to control the interface display and computational steering. At the moment there is no means to incorporate the model description (functions) in the XML format, although eventually 
Table 1. Main XML tags used in PhysioML

\begin{tabular}{|l|l|l|}
\hline Display & & User Interface Definition \\
\hline & panel & defines a window panel \\
\hline & image & URL for screen image \\
\hline & label & screen display \\
\hline & & \\
\hline Model & & Model Definition \\
\hline & variable & define variable (name, initial value) \\
\hline & transfer & transfer matrix \\
\hline & box & define a compartment \\
\hline & boxstuff & image displayed in compartment \\
\hline & boxtrigger & threshold for compartment \\
\hline
\end{tabular}

it is envisioned that this can be accomplished using MathML. For details on

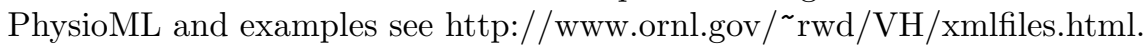

\section{Virtual Soldier}

The purpose of the DARPA VS Project, started in 2004, was to use physiological models and data to predict the location of a wound to the heart (left ventricle or right ventricle) caused by a fragment. The specific examples modeled included small fragments wounds to myocardial zones 7 and 12 of the left ventricle with the medical consequences being either tamponade or exsanguination. While different software was used to implement the computational framework for VS and more sophisticated models were utilized, many of the concepts for the VS, especially for the user interface, extrapolate concepts originally developed for the $\mathrm{VH}$.

\subsection{The VS Holomer Concept}

An important concept developed in the VS Project was that of the Holomer. The Holomer incoporated both the computational framework, including the data and properties (molecular, biochemical, cellular, physiologic, organ, tissue and whole body), computational models, and the display environment. The focus of Phase I was on the heart, so the anatomy considered in the VS Holomer was restricted to the heart and surrounding major vessels.

\subsection{Computational Framework for Phase I}

For Phase I two types of computational modeling were conducted: 1) high-level integrative physiological (HIP) models (circuit models) and 2) three-dimensional finite element (FE) models, including electrophysiology and mechanical motion. The HIP models were optimized to the physiological characteristics and results were then passed, via file transfer, to the FE models.

The results of both types of computations were integrated using a visualization environment based on SCIRun [7. In addition, visualization using the 
SCIRun environment was linked to the VS Knowledge Base (VSKB), an ontology containing definitions for anatomical terms (the Foundational Model of Anatomy) and physiology. The project also developed an XML format for describing the fragment wound. The integration of output using SCIRun was what was generally referred to as the Holomer.

ORNL and its partner, the Center for Information Technology at the University of South Carolina, developed two components of the VS computational framework. First, we developed middleware to support the project including Web services for the data repository, a client to connect to the Web service for the VSKB, and services and associated client API for the HIP model computations. The University of Washington developed a Web service for the Foundational Model of Anatomy [6]. These middleware components, various Web services for data repository, computations, and ontologies, provide a good infrastructure for a future comprehensive computational framework. Unlike the Phase I framework, this infrastructure would facilitate launching computations from the environment.

The second component of the work conducted at ORNL involved the development of a "HotBox" within the SCIRun visualization environment. The "HotBox" facilitated interaction between the VSKB ontologies (specifically the anatomical ontology) and the geometric anatomical models and between the anatomy and associated physiology (see Fig. 2). The problem was to display

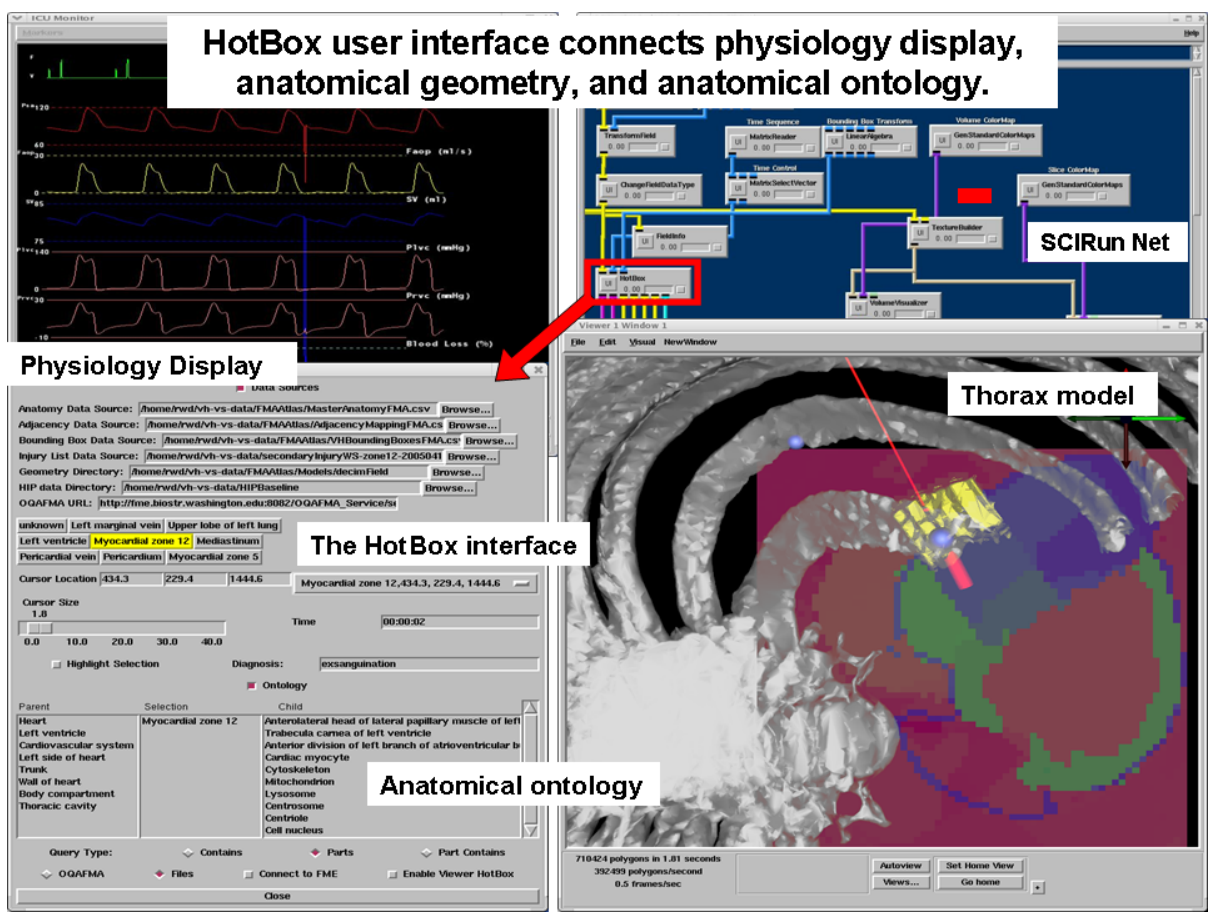

Fig. 2. Virtual Soldier Holomer User Interface 
this information in such a way as to capture the three-dimensional (3D) nature of the human body and to correlate that with extensive information about both the anatomy and the physiology of the wounded soldier [5]. The VS "HotBox" succeeded in providing this connectivity.

\section{Future}

In the future we should begin to see true integrative environments for multiscale human modeling and simulation. A major challenge is to integrate two very different modeling approaches, one based on discrete information (e.g., stoichiometric biochemical reactions) and one based on continuous, time-dependent simulation (e.g., differential equation-based systemic organ models). Two concepts are suggested as potential ways to bridge these different modeling approaches.

1. Layering of information. This involves the use of autonomous agents to enable knowledge discovery in support of modeling and simulations. This knowledge discovery process can mine what is known about relevant anatomic, metabolic, or physiological information that impact simulations.

2. Discrete-event simulation (DEVS). Discrete event simulation is being used to model continuous simulations [4]. Since DEVS by definition can also incorporate discrete reaction kinetics, it has the potential to provide the necessary bridge to bring about integration between these approaches. A conceptualization of our future vision is contained in Fig. 3.

In the concept vision, the ontological information layers (obtained both by existing libraries of information and supported by intelligent agent searches) support a computational layer based on a combination of DEVS and continuous

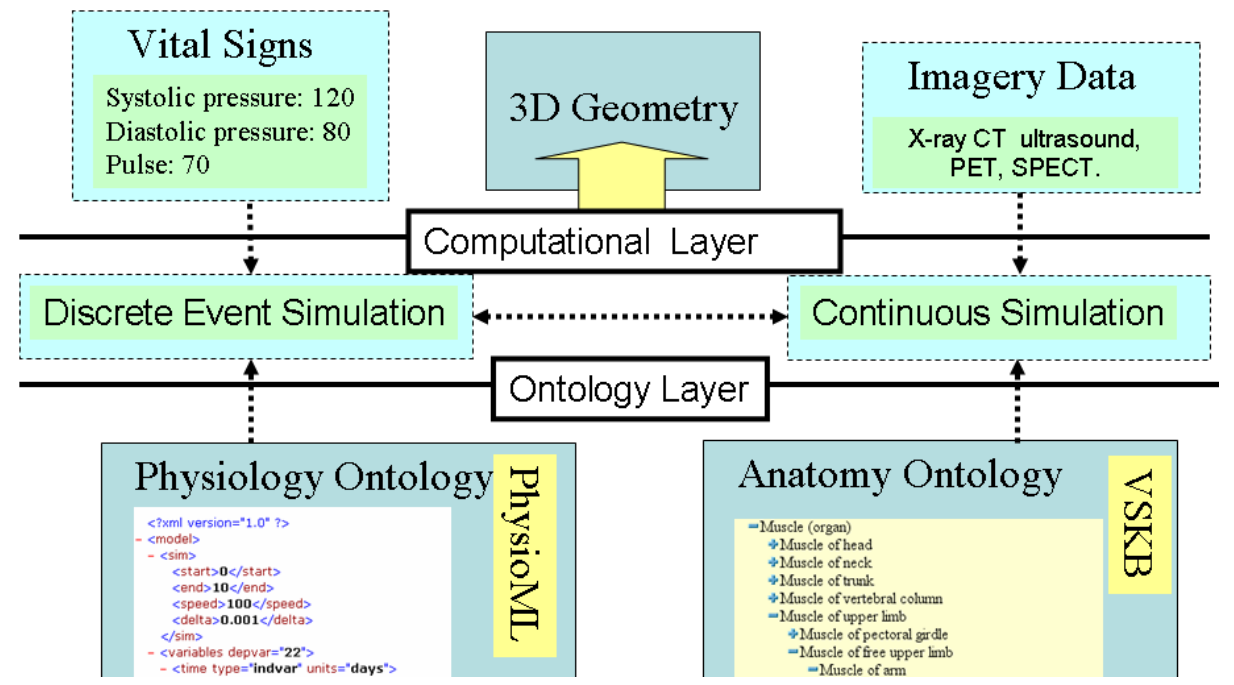

Fig. 3. Future vision of the Virtual Human or Digital Human Infrastructure 
simulation approaches, which in turn supports the data and user interface layers. This concept uses capabilities and characteristics developed in the VH and VS projects.

DEVS has a performance advantage over continuous simulation which can be understood intuitively as follows. The time advance function determines the frequency with which state updates are calculated at the spatial grid points. The time advanced at each grid point is inversely proportional to the magnitude of the time derivative at that point, and so "regions with slow change will have large time advances relative to regions that are changing quickly. This causes the simulation algorithm to focus effort on the changing portion of the solution, with significantly less work being devoted to portions that are changing slowly" [4.

Our suggestion is that DEVS can be used to implement multiscale computations when there is loose coupling between "fast" states and "slow" states of the model system. DEVS can incorporate both discrete models (such as stoichiometric chemical reactions) and continuous models (organ systems) to provide a comprehensive computational framework for the Digital Human.

\section{Summary}

We have presented a brief historical account of development of integrative human modeling, using the VH Project and the DARPA VS Projects as examples. The lessons learned from these examples and the successes attained have been discussed. In addition, we have outlined the difficulties faced by these projects in attaining a truly integrated human modeling and simulation environment. Finally, we have addressed what we believe to be the future in this effort, the push to attain fullyintegrated, multiscale modeling, incorporating both discrete metabolic reactions and continuous modeling based on differential equation models.

We have proposed two different capabilities which might overcome some of these difficulties and make possible fully integrative modeling. These are: 1) layering of information and 2) discrete-event simulation. Each of these concepts are discussed and their usefulness to human modeling and simulation outlined. We believe that enormous strides will be made in the coming years toward fully-integrative human modeling, just as scientists have made similar strides in climate modeling over the last decade. By breaking down the barriers between discrete models and continuous models, we believe that the goal of a truly integrated computational environment for human modeling will be a reality in the not-too-distant future.

\section{References}

1. S. P. Dickson, L. C. Pouchard, R. C. Ward, G. Atkins, M. J. Cole, B. Lorensen, and A. Ade. Linking Human Anatomy to Knowledgebases: A Visual Front End for Electronic Medical Records. In Medicine Meets Virtual Reality-13 Conference Proceedings. IOS Press, 2005.

2. C. Krause. The Virtual Human Project: An Idea Whose Time Has Come? ORNL Review, 33(1), 2000. 
3. C. S. Lindsey, J. S. Tolliver, and T. Lindblad. JavaTech, an Introduction to Scientific and Technical Computing with Java. Cambridge University Press, Cambridge, UK, 2005.

4. J. Nutaro. Discrete event simulation of continuous systems. To appear in Handbook of Dynamic Systems Modeling, 2005.

5. L. C. Pouchard and S. P. Dickson. Ontology-Based Three-Dimensional Modeling for Human Anatomy. Technical Report ORNL/TM-2004/139, Oak Ridge National Labortory, 2004.

6. C. Rosse and J. L. V. Mejino. Ontology for bioinformatics: The foundational model of anatomy. Journal of Biomedical Informatics, 36:478-500, 2003.

7. Scientific Computing and Imaging Institute (SCI). SCIRun: A Scientific Computing Problem Solving Environment, 2002.

8. R. C. Ward, K. L. Kruse, G. O. Allgood, L. M. Hively, K. N. Fischer, N. B. Munro, and C. E. Easterly. Virtual Human Project. In Proceedings of the SPIE: Visualization of Temporal and Spatial Data for Civilian and Defense Applications, pages 158-167, 2001.

9. R. C. Ward, D. J. Strickler, J. S. Tolliver, and C. E. Easterly. A Java User Interface for the Virtual Human. In Proceedings of the Joint BMES/EMBS Conference, page 1211, Atlanta, GA, 1999. 